\title{
UNIVERSIDAD Y POLÍTICA: \\ La universidad de San Marcos en la segunda mitad de los noventa ${ }^{1}$
}

Omar Yalle Quincho

\section{Resumen}

El presente trabajo tiene por objeto, aproximarnos al contexto generacional estudiantil de los noventa, conocer cuáles fueron las principales organizaciones políticas de izquierda que actuaron, los acontecimientos que se dieron en ese lapso de tiempo, así como los logros alcanzados.

Palabras clave: estudiantes, movimiento estudiantil, organizaciones políticas, socialismo/comunismo, universidad.

\section{Abstract}

This paper aims, approach the student generational context of the nineties, to know what were the main leftist political organizations that act, the events that occurred in that period of time as well as achievements.

Keywords: student, student movement, political organizations, socialism/communism, University.

El presente trabajo es un extracto de la tesis intitulada: "La cultura política en el discurso de los dirigentes universitarios de la izquierda sanmarquina: 1995-2000", presentado a la Universidad Nacional Mayor de San Marcos para optar el Titulo Profesional de Licenciado en Antropología el año 2008. 


\section{El contexto generacional}

La generación de los noventa se caracterizó hasta cierto punto por vivir aislada de los problemas de la realidad nacional y universitaria. En la Universidad Nacional Mayor de San Marcos encontramos un movimiento estudiantil en ciernes, las iniciativas colectivas se dan con menor frecuencia y serán más de aglutinamiento por causas universitarias y nacionales de coyuntura, que de articulación y organicidad y/o por la reconstrucción de sus organismos de presión legítimos como los centros de Estudiantes, Centros Federados y la Federación Universitaria de San Marcos². Estuvo ausente la discusión ideológica y las propuestas políticas. Ya a fines de los noventa, con el agotamiento del Estado corporativo y la inviabilidad del sistema económico, es que se toma conciencia y la universidad conjuntamente con el pueblo se ve en la necesidad de salir a las calles y expresar su disconformidad y rechazo, aunque no precisamente de manera institucionalizada ${ }^{3}$.

Los jóvenes sanmarquinos ya no vivirán la política como los estudiantes de las generaciones anteriores inmediatas, es decir, el de una militancia comprometida con un proyecto, ideológicamente de transformación, sino, desde la lucha reivindicativa y el juego de la democracia burguesa. Esto explica, porque ni bien terminada las funciones de la Comisión Reorganizadora en la Universidad y el gobierno fujimorista en el año 2000, hay ausencia de debates y propuestas en ese sentido. Esto explica, pues, que durante la intervención se dio importancia a lo político respecto a lo ideológico, primó la acción sobre la discusión teórica. Por lo tanto hay una juventud que le rehuye al compromiso militante.

Los cambios neoliberales en la educación peruana aperturaron un nuevo debate en relación a la Universidad: gratuidad de la enseñanza, calidad académica, estructura académico-administrativa, entre otros aspectos. Se aplicó una política antiestudiantil de característica económico-social; el pago por diversos conceptos que se fueron incrementando: matriculas, certificados, constancias, examen de admisión, centro preuniversitario. La Universidad empezó un proceso de elitización, se hizo difícil articular el movimiento estudiantil. Las expresiones de unidad y frente, las coordinadoras y las asambleas de gremios y estudiantes, son respuestas sin mayor trascendencia frente a los problemas que interpretaban la mayoría de estudiantes. Las grandes utopías que movieron a la izquierda en los setenta y ochenta pasan a un segundo plano. La trasformación de la sociedad no pasa ya necesariamente por el universo ideológico de las propuestas marxistas.

Creemos necesario mencionar algunas características sociales en los estudiantes de los noventa en la Universidad Nacional Mayor de San Marcos: el apoliticismo

2 Con la inclusión legal del Tercio Estudiantil en la ley universitaria de 1983 y el estatuto de la Universidad Nacional Mayor de San Marcos de 1984, se crea un paralelismo de representación, neutralizando de alguna forma, la acción directa de los estudiantes y el fortalecimiento de su representación gremial.

3 Al respecto puede revisarse el trabajo de Deborah POOLE, y Gerardo RENIQUE: Movimiento popular, transición democrática y caída de Fujimori, en: Memoria 147, México, 2001. 
como hastío de una actividad desprestigiada que a partir del discurso neoliberal logra una opinión favorable en la población; el individualismo como expresión económica y social de los sectores medios desplazados que llegan a la universidad con nuevos códigos de comportamiento y consumo — esta canalización se dio a través del centro preuniversitario motivando la marginación de los sectores populares, del derecho a la educación-; la desmovilización que se tradujo en la inacción del nuevo componente social que no veía en la actividad gremial y política una necesidad concreta de afianzamiento de la vida universitaria. Por otro lado, la institucionalización del clientelaje, la prebenda, la corrupción y la mediocridad, en quienes gestionaban la Universidad. En esencia se impuso la ideología de la antipolítica, expresada, como consecuencia del predominio de la violencia política, la lógica de mercado, la crisis económica y el cambio en la composición social del estudiantado sanmarquino ${ }^{4}$.

En los noventa, en la Universidad Nacional Mayor de San Marcos, se dan las condiciones para expresiones culturales que disienten del discurso y de la práctica de generaciones anteriores que expresan nuevas formas de pensar y hacer política. En ese sentido fue educada la generación de la primera parte de los noventa. Se afirma el terreno para cultivar el descrédito de la política, generando las condiciones propicias para una intervención militar y administrativa, que tuvo como justificación la corrupción y como pretexto, la subversión. El sentido de la crisis de la universidad, también lo encontramos en las bases sociales y políticas que la sustentaron.

a) Bases sociales

\section{Movilidad social inversa}

Una de las explicaciones que sustentaron el hecho político más importante en el claustro durante la segunda mitad de los noventa, como fue la Comisión Reorganizadora, lo encontramos en el cambio de la composición económico-social del estudiantado. Los jóvenes que accedían a la Universidad Nacional Mayor de San Marcos entre fines de los cincuenta y fines de los ochenta, provenían principalmente de los sectores populares de Lima y de diversas provincias del país, consecuencia de la explosión demográfica en Lima y el litoral costeño. En la década de los setenta, la cantidad de provincianos que ingresaban a San Marcos superaba el $40 \%$ del estudiantado. ${ }^{5}$ Estas cifras han disminuido considerablemente desde fines de los ochenta. La juventud de los sectores populares vio restringida su posibilidad de seguir estudios universitarios. Muchos optaran por trabajar para sobrevivir. Aun así, se sabe que alrededor del $75 \%$ de los estudiantes universitarios sanmarquinos provienen de escuelas públicas, se observa que la mayoría son nacidos en la ciudad de Lima, sigue siendo

\footnotetext{
4 Para una mejor explicación véase PONCE, Grover: El discreto encanto del desencanto. Jóvenes, política y régimen fujimorista en San Marcos de los noventa, Mimeo, Lima, s/f, pp. 2-11.

$5 \quad$ Al respecto véase Nicolás LYNCH: Los jóvenes rojos de San Marcos. Lima, El zorro de abajo, 1990.
} 
mayoritariamente de origen popular, y su visión de la Universidad y del país ya no es necesariamente provinciana como en los sesenta y setenta ${ }^{6}$.

La educación superior universitaria era una opción cada vez más difícil de realizarse. El cambio del modelo económico a inicios de los noventa también restringió las posibilidades de la clase media venida a menos, que desplazadas de las universidades particulares, accedían a las universidades públicas “... las clases sociales se han pauperizado, y por lo tanto, sus expectativas de seguir una carrera profesional; los estudiantes de las clases más acomodadas han ido descartando paulatinamente su voluntad de estudiar en una universidad particular" ${ }^{7}$. Esto ha originado un proceso de movilidad social $^{8}$ descendiente tanto de los sectores menos favorecidos - las clases popularescomo de los sectores de clase media, fundamentalmente limeña. El sanmarquino de los noventa es un estudiante con un nuevo perfil, otro en relación a décadas anteriores:

... aunque aún yo no me explico, estoy viendo tanto en sociales como en toda la universidad, como se esta produciendo un fenómeno de elitización en sus ingresantes. Ahora, ingresan cada vez un número mayor de estudiantes de la Universidad Católica, mientras que antes había uno o dos por promoción. A esa gente se le saca inmediatamente por su manera de comportarse, de hablar, de vestir. Son bien diferentes 9 .

\section{Crisis de identidad}

La identidad generacional la podemos definir como los rasgos de una colectividad que se caracteriza por ciertas actitudes y condicionamientos comunes a todos aquellos que convienen en un momento histórico determinado. ${ }^{10}$ Una generación es diferente a otra, en la medida que sus códigos, símbolos, identidades e imaginarios suelen por lo general ser disímiles:

En el viejo paradigma las identidades colectivas están en función de códigos socioeconómicos e ideológico-políticos (estudiantes,

\footnotetext{
6 Algo que caracterizó a los estudiantes de provincias en la década de los setenta, era su cultivo por la identidad peruana que se va a expresar en el folklore desde la música y las danzas. Posteriormente, en los ochenta y noventa optaran por otros géneros musicales como la salsa, el rock y el pack.

$7 \quad$ Los cachimbos, la política y San Marcos. Los muchos cambios de los noventa, en: Cuestión Previa, Lima, N" 2, junio 1991, p. 25.

8 La movilidad social es una categoría de las Ciencias Sociales que nos permite analizar como las personas o grupos de personas se desplazan progresivamente de posición con respecto a su clase social de manera ascendente o descendente perdiendo determinados roles y status y/o adquiriendo otros nuevos.

9 Entrevista a Felipe Bravo Navarro, en: Cuestión Previa, Lima, N” 2, junio 1991, p. 28.

10 MALPARTIDA, Víctor y Luís HOCES: Encontrando el horizonte utópico. La esperanza jodida de los jóvenes. Lima, Grafi Perú EIRL, 1992, p. 66.
} 
jóvenes urbano populares, socialistas, etc.). En el nuevo paradigma, las identidades son construidas en relación a espacios de acción y mundos de vida como: género, preferencia sexual, sobrevivencia de la humanidad (medio ambiente), derechos indígenas, de las mujeres, democráticos, etc. Se plantea una ética global en cuanto a las relaciones con la naturaleza, la relación entre los géneros, la relación con el cuerpo, las relaciones entre los individuos, la importancia de la subjetividad, la tolerancia y la solidaridad. ${ }^{11}$

El movimiento estudiantil ha puesto en practica otras alternativas de participación vinculadas al ejercicio de la ciudadanía como; los movimientos ecologistas, feministas, de contracultura, etc., que constituyen nuevas formas de expresión del movimiento social, a diferencia del clásico movimiento de obreros, campesinos y donde el movimiento estudiantil tenia también un alto grado de organización y politización. ${ }^{12}$ En la actualidad, el movimiento estudiantil parece, empieza a perder espacios y se inserta en un contexto mayor; la sociedad, sin perder su identidad. Pareciera que estuviéramos ante la desaparición de una cultura estudiantil homogénea vigente hasta los setenta, como lo ha observado José Joaquín Brunner para el caso latinoamericano, por la sustitución de una diversidad de culturas estudiantiles producto de la heterogeneidad de las clases sociales y de las preocupaciones diferenciadas de estos. Brunner anticipó un comportamiento de los estudiantes menos interesados en convertir la institución universitaria en una partera de la modernidad y en cambio ve el predominio de movimientos estudiantiles diversificados y preocupados por la defensa de intereses gremiales y corporativos ${ }^{13}$.

Hoy en día la identidad sanmarquina se encuentra fragmentada; distintos códigos y símbolos parecen separar a los estudiantes de una facultad a otra, de un colectivo y/o agrupación de otras similares. Hay nuevos intereses, nuevas perspectivas, como que el movimiento estudiantil se hubiera diversificado, pero a la vez parece conservar su unidad, al menos en los problemas generales. Los problemas son diferentes, la atención y prioridad frente a ellos también lo son. Los estudiantes son

11 KRAUSKOPF, Dina: "Dimensiones críticas en la participación social de las juventudes", en: Sergio BALARDINI: La participación social y politica de los jóvenes en el horizonte del nuevo siglo. Buenos Aires, CLACSO, 2000, p. 129.

$12 \mathrm{Al}$ respecto puede revisarse el texto de Anthony BEBBINGTON, Martín SCURRAH y Claudia BIELICH: Mapeo de Movimientos Sociales en el Perú Actual. Lima, CEPES, 2008. También CASTELLANOS, Themis; CARAMUTTI, Rosalía y Mercedes UBILLUS: Lo viejo y lo nuevo de la acción colectiva en Lima: experiencias de participación ciudadana en Lima. Lima, Centro ALTERNATIVA, 2003. Así mismo, para entender el desarrollo evolutivo de los movimientos universitarios en relación al movimiento social hasta inicios de los setenta, puede revisarse el texto de Enrique BERNALES: Movimientos sociales y movimientos universitarios en el Perú. Lima, PUCP, 1974.

13 GROMPONE, Romeo: El velero en el viento. Sociedad y política en Lima. Lima, Instituto de Estudios Peruanos, 1991, p. 40. 
socialmente heterogéneos y en comparación a décadas pasadas parece no importarles las distancias. Es como si el gran continente sanmarquino se hubiera partido en un inmenso archipiélago, cada uno dispuesto a afirmar su identidad ${ }^{14}$. Hay preocupaciones y realidades heterogéneas y dispersas que reconocen a algunos e ignoran a otros. Pareciera ser que lo que fue de San Marcos el tiempo se lo llevo y que el viejo discurso integrador resultaría ya obsoleto.

\section{Pragmatismo}

Más allá de las reflexiones teóricas y de los cambios en las perspectivas y proyectos de los jóvenes universitarios, existió una clara convicción de lo inmediato y utilitario, de lograr determinados objetivos dentro del sistema económico, político y social, lo que se concreta en el ser profesional, en la aceptación del grupo social y en la adquisición de un estatus dentro de la sociedad.

Al no existir referentes políticos e ideológicos, se busca una identidad personal, el yo como medio de superación y realización. Una adecuación al modelo, al sistema, y ya no en la posibilidad que le otorgaba el mito de trasformar la sociedad. El asumir su yo personal se vuelve gravitante. La mayoría de estudiantes quieren apropiarse del conocimiento, ser tratados como ciudadanos, sus proyectos se traducen de manera individual y no de manera colectiva. El nuevo sanmarquino pasa menos tiempo en la universidad, el nuevo contexto de crisis le exige ser competitivo, a actuar de manera personalizada y perder de vista las ilusiones y acciones colectivas:

... el ámbito de la persona, y lo relacionado a la vida misma, la búsqueda ya no de un ideal abstracto que negaba el presente y niega por completo el derecho a la vida, a una vida privada, sino aquel que se afirma en uno mismo. Hoy la persona ha dejado de ser exclusivo del fuero interno como la iglesia, la familia y/o el partido. Los jóvenes universitarios han indagado y asumido su yo personal y fortalecido la necesidad de autoestima personal ${ }^{15}$.

A diferencia de los sectores populares que se educan en las universidades nacionales, la educación de los sectores exclusivos y de la elite dominante, tienen una visión liberal; funcional y tecnocrática de la universidad, donde la eficiencia de sus egresados es vital para su inserción en el aparato productivo y de servicios. Por el contrario, el modelo de educación y particularmente de universidad, debiera más bien, generar una industria para el desarrollo nacional. En tal sentido es viable alentar la forja de una Universidad Nacional, Científica y Democrática. Ahí la tarea de los estudiantes de las nuevas generaciones.

\footnotetext{
14 MEJIA, Carlos: “Silencios y sonrisas en los pasadizos", en: Herejes y Renegados, segunda etapa, N. ${ }^{\circ}$ 14, agosto-septiembre, Lima, 1995, p. 5.

15 MONTOYA CANCHIS, Luís: “Los varios colores de la juventud universitaria”, en: Revista de Sociología, Vol. 8, N" 9, UNMSM, Lima, 1994, p. 145.
} 
b) Bases políticas

\section{Fin de las ideologías}

La caída del Muro de Berlín y la dessestructuración de la URSS, repercutió ideológica y políticamente en las organizaciones de la izquierda peruana. Se hicieron muchas lecturas en torno a la crisis, que se tradujeron en el cuestionamiento y el replanteamiento de las propuestas por parte de los jóvenes:

la opción política de los jóvenes ya no se guiará propiamente por referentes ideológicos, lo que resultara más bien serian las necesidades derivadas de la actividad universitaria. Es por ello que se pueden registrar el reemplazo de las opciones políticas de los grupos de izquierda por los movimientos independientes ${ }^{16}$.

Hay una reorientación de los discursos. El estudiante sanmarquino ya no es el sujeto revolucionario que lo quiere cambiar todo desde la universidad, sino un activista de la coyuntura, sin grandes mitos. Sin embargo, creemos, teniendo en cuenta esta realidad, que las ideologías y formas de hacer política desde la perspectiva socialista siguen vigentes y se seguirán transmitiendo de generación en generación. ¿Por qué?, primero, el mito integra las sociedades y grupos humanos, proveyéndoles identidad y funcionalidad, y dos; la justicia social es un problema pendiente que es alimentado desde las ideologías, particularmente de izquierda.

\section{Desencanto y escasa participación politica}

La actividad política, aquella que involucra las relaciones humanas y la practica en torno a la conquista del poder es vista como un camino que no conduce a nada, un proceso recurrente que linda con los valores. El sanmarquino de los noventa asociaba la universidad con la política en un sentido negativo. A los jóvenes de los noventa que no les interesa la política, buscan otros espacios a través de los cuales puedan realizar sus proyectos colectivos como las actividades religiosas, la proyección social y otros espacios alternativos en la universidad considerados como no políticos (eventos deportivos, culturales y artísticos, etc.). Este anhelo de comunidad en los sectores universitarios, se explica por el escepticismo político, la falta de referentes en términos de expectativas y las frágiles esperanzas que concita la educación superior ${ }^{17}$.

Otra explicación de la deslegitimación de la política, lo encontramos en la "extrema radicalidad en el discurso" que llevaba a un divorcio de la realidad y los intereses estudiantiles, sumado al desarrollo de practicas clientelistas, que priorizaban

16 MONTOYA, Luís: Nido de inquietudes. Universidades y jóvenes: politica de desarrollo universitario, actores sociales y modernidad educativa, Tesis para optar el Título Profesional de Licenciado en Sociología, Lima, UNMSM, 1993. p, 180.

17 GROMPONE, Romeo: op cit., p. 54. 
el interés de grupo antes que el de la mayoría de estudiantes, creando el descrédito de las organizaciones y de sus representantes ${ }^{18}$.

El típico sanmarquino de los noventa, es indiferente. Se caracteriza entre otras cosas; por una marcada preocupación académica, por un rechazo abierto a toda política partidaria, su escepticismo a la reivindicación gremial y un abstencionismo en la participación de movilizaciones y procesos electorales. Ellos constituyen la mayoría de la población universitaria en San Marcos y la base social pasiva del fujimorismo y del neoliberalismo. Esta nueva generación de estudiantes legitimará las medidas del gobierno hacia la universidad, como la intervención militar en Mayo de 1991 y la intervención administrativa en Mayo de 1995. La indiferencia, es una politización negativa, un estado de ánimo, que aparenta neutralidad:

... la indiferencia como actitud política del estudiante de base, no es apoliticismo, ni es una despolitización a fondo como comúnmente señalan algunos analistas, sino es una politización negativa, es decir, es una actitud política que expresa todo un estado de animo, aparentemente de neutralidad $\mathrm{e}$ indiferencia frente a la esfera publica ${ }^{19}$.

Es una manera conservadora de tomar una posición frente a la universidad y los problemas del país ${ }^{20}$. En este sentido; “... el terror, la militarización, la demagogia y la crisis de partido y liderazgo, fueron ingrediente necesarios para la inauguración de una nueva era; la de la antipolítica. Para ello fue indispensable, quebrantar identidades, disolver la moralidad publica, reducir las expectativas de la juventud y mutilar la conciencia social"'21.

\section{Las principales organizaciones políticas}

Las principales organizaciones políticas estudiantiles que estudiamos en la segunda mitad de los noventa, son espacios emergentes, exclusivamente universitarios, más o menos institucionalizados, legitimados, que no tienen por objeto la transformación social, sino la búsqueda, desde la lucha política, de la democracia y la garantía de la libertad y de los derechos civiles, políticos y económico-sociales, así como la lucha por la vigencia plena de los derechos estudiantiles. Las organizaciones estudiantiles, por lo general, son núcleos diversos y variados como círculos de estudios, talleres, grupos de revista de opinión, colectividades políticas independientes, organizaciones culturales,

18 MONTOYA, Luis: "Las otras elecciones. San Marcos: la otra mayoría”, en: Revista Quehacer, N“80, Desco, Lima, 1992, p. 40.

19 PONCE, Grover: op cit., p. 19.

20 GROMPONE, Romeo: op cit., p. 18-19.

21 VARGAS, Moisés: La violencia política en la escena sanmarquina: 1990-2000, mimeo, Lima, 2002. 
organizaciones religiosas, listas electorales, etc. Lo que une a la gran mayoría de ellas, es la negación a la forma clásica de hacer política, a las formas de asumir la política tradicional2.

Hay un espíritu de negación a la experiencia política anterior. Sin embargo son estas nuevas identidades que se van a expresar con nuevos símbolos y discursos. También hay una creciente preocupación por los problemas inmediatos, coyunturales y recurrentes. Estas organizaciones por lo general serán de carácter universitario y temporales. Sin embargo, las organizaciones políticas estudiadas, tienen referentes históricos, políticos e ideológicos, lo que no niega su continuidad con otros nombres en un nuevo contexto. En la Universidad Nacional Mayor de San Marcos todas las tendencias tendrán su espacio para la discusión y organización. Es en este periodo donde se forjaría una nueva identidad de la izquierda universitaria sanmarquina.

Las organizaciones políticas de izquierda en la Universidad Nacional Mayor de San Marcos de los noventa, en cierta forma, son herederos de la historia y tradición de partidos políticos que la antecedieron, quienes a través de algunos líderes, habrían inspirado su origen y constitución en esta década, pero diferenciándose estas nuevas como estudiantiles, con presencia universitaria e identidad propia. Cabe indicar como lo ha señalado uno de los dirigentes: todas estas organizaciones tienen su pasado bistórico, ${ }^{23}$ han modificado sus identidades para seguir activos en la dinámica universitaria y hacer receptivo el discurso durante la segunda mitad de los noventa.

Es en ese panorama que la izquierda universitaria asume el rol de nueva cultura política generacional, donde el apoliticismo será una constante en el devenir estudiante. Surgen las organizaciones, como una respuesta a la llamada generación X, generación del tiempo perdido, sin aspiraciones e ideales, conservadora, que se manifiesta indirectamente en concordancia con los propósitos del Estado y las autoridades universitarias, de mimetizar y desmovilizar a los estudiantes. Entre las principales organizaciones políticas estudiantiles a mediados de los noventa tenemos: Integración Estudiantil, Juventud Popular, el FER-SM y el Colectivo Amauta.

a) Integración Estudiantil y la construcción de un movimiento socialista en el país

Integración Estudiantil surge en abril de 1998 como parte germinal de un proyecto político común, de una juventud que apostaba por una izquierda independiente. Se definen como una organización que se afirma por el socialismo, pero diferenciándose de la izquierda tradicional. Realizan una labor gremial en la Universidad y trabajan con sectores estudiantiles y obreros fuera de ella. Señalan que será necesaria la confluencia con otros sectores para la forja de la unidad. Se afirman en las propuestas

\footnotetext{
22 PONCE, Grover: op cit., pp. 16-17.

23 MIRANDA, Oscar: "San Marcos. Detrás del Che se esconde Mao. Radicales en la sombra”. en: Domingo, La República, N. ${ }^{\circ}$ 213, Lima, 30 de junio de 2002.
} 
revolucionarias, donde los compañeros coincidan en posiciones ideológicas similares y en torno a la problemática universitaria y bajo nuevas formas de hacer política. En el plano ideológico, se adhieren al pensamiento del Amauta José Carlos Mariategui y el legado de Ernesto Che Guevara, lo que los identificaría ante las demás opciones como una organización guevarista:

Nos formamos el año 98, a mediados de año, entendiendo que los problemas que afronta la educación y en general la sociedad peruana no pasan simplemente por el problema de las reivindicaciones estudiantiles, sectoriales, sino, entendiéndolo como un problema político mayor. Ahí vemos la necesidad de afrontar eso, desde formas orgánicas políticas. Así nació Integración Estudiantil. Entonces en esos años; 98, 99, es que nosotros estábamos viendo el perfil de la organización que se estaban construyendo, porque Integración Estudiantil nace básicamente como una organización estudiantil de compañeros que se afirmaban en la izquierda, en el socialismo, pero que disentían o no estaban identificados con la práctica de las organizaciones tradicionales de izquierda.

Luego de esos dos años empieza a haber una mayor claridad, con los compañeros de la organización, y empieza un trabajo por fuera, también de la actividad gremial reivindicativa. Es ahí, donde empezamos a desarrollar talleres, proyectos, debates políticos, electorales, sobre la vigencia o no del socialismo, sobre la coyuntura política y a entrar al debate. Consideramos que Integración Estudiantil es una organización que necesariamente va a confluir con otras organizaciones y núcleos que han ido surgiendo en los últimos años y a la vez reafirmarse en una propuesta de izquierda revolucionaria, de articular organizaciones nuevas y marcar un distanciamiento frente al retroceso o desfase que tienen las organizaciones tradicionales de la izquierda peruana.

... en el plano ideológico y político nosotros nos definimos como una organización socialista, bajo el legado básicamente de José Carlos Mariategui y del Che Guevara, ¿porqué?. Uno; fundamentalmente en términos ideológicos, porque lo consideramos como el fundador del movimiento socialista en el Perú e incluso en América Latina y creemos que a partir de su pensamiento podemos reactualizar la visión marxista de la realidad peruana y de su desarrollo. Dos, en cuanto al che, porque tuvo que aportar dos cosas principales: uno; en el tema de la ética de la militancia, dos: la coherencia entre el discurso y la práctica, que es algo que lamentablemente muchos sectores de la izquierda tradicional han abandonado y lo que queremos es básicamente una izquierda revolucionaria. No estamos, no creemos que el 
camino por conquistar el socialismo pase por una cuestión exclusiva de aplicar reformas a partir del Estado burgués"24.

b) Juventud popular, un movimiento amplio

Juventud Popular en una organización que nace en 1995 como parte de todo un proceso de articulación de fuerzas y espacios incluyendo el estudiantil. Se definen como una organización antineoliberal y antidictatorial, que se diferencia de la denominada generación X, llamada así a la generación de los noventa, por carecer de grandes sueños y mitos.

Juventud Popular es una organización de carácter nacional. Entre sus principales acciones, se van a manifestar en contra de la privatización de las empresas públicas, en especial en el caso de Petro-Perú. Llevaran a cabo una acción cívica importante con otros sectores, recolectando firmas para llevar a cabo un referéndum, donde el pueblo decida el destino de la empresa estatal. Participarán activamente en la organización de las movilizaciones del 4 y 11 de junio de 1998. Desenvuelven un trabajo a nivel universitario y coordinan acciones conjuntas con los sindicatos. Algunos sectores políticos han calificado a Juventud Popular de ser la Juventud del PCP Patria Roja ${ }^{25}$, organización con una fuerte incidencia en el sector magisterial y estudiantil en la década de los setenta y ochenta:

Juventud Popular nace en diciembre del 95. Hay una reunión nacional del 2 al 5 de diciembre de estudiantes y jóvenes, de grupos barriales, donde estaban; Joven Pueblo, que era básicamente del Cono Sur y sectores del Cono Norte de Lima, dirigentes estudiantiles, me refiero gremiales y gente del FER del Perú. Ellos conforman Juventud Popular, como un movimiento amplio, nacional, democrático, patriótico y progresista, que busca organizar a los jóvenes para concientizarlos, para que luchen por una transformación en el país.

Salimos como una organización que quería también romper con ese estigma que nos metían a los jóvenes como la generación X, la generación pragmática, que no nos importaba nada más que nuestra individualidad. Luchábamos porque la gente se organice. En el 98 impulsamos esa movilización nacional juvenil ${ }^{26}$ por ser también un acuerdo congresal del encuentro nacional. En el encuentro nacional se aprueban unos lineamientos del programa. Programas son las

$24 \quad$ Integración Estudiantil.

25 Escisión del Partido Comunista del Perú-Bandera Roja de tendencia pro-China que surge a partir de las discrepancias con el sector de Saturnino Paredes y que forman una organización propia el año 1968.

${ }_{26}$ Se refiere a la movilización convocada por el Comité Cívico-Patriótico como se le denominó a la jornada de protesta del 4 de junio de 1998. 
banderas que nosotros levantamos ante el pueblo acerca de lo que nosotros proponemos para el país.

Juventud Popular empieza su lucha contra la privatización de Petro-Perú, recolectando firmas para el referéndum, para que no se privatice. Luego pasada la lucha por el referéndum, se asumió la lucha contra la reelección de Fujimori. En el 97 llevamos a cabo un encuentro nacional, en el cual se define a Juventud Popular como una organización antidictatorial y antineoliberal. En el encuentro nacional del 97, se da esa unidad de criterios entre todas las bases que se habían estado formando. Éramos ocho departamentos al inicio, y después fuimos creciendo y era necesario dar una unidad de criterios, entonces se da el encuentro nacional, en que se define antineoliberal, antidictatorial, y por el compromiso de luchar por la reconstrucción y reorientación de la FEP, que es el máximo gremio estudiantil y es el sector donde tenemos mayor influencia política ${ }^{27}$.

c) El FER-SM y la premisa del frente único

El FER-SM es tal vez la organización con más tradición e historia política dentro de la Universidad Nacional Mayor de San Marcos. Desde sus inicios en 1958, se define como una organización estudiantil de carácter revolucionario. En un primer momento, agrupó a estudiantes de izquierda progresista, democrática y revolucionarios independientes, sin filiación política partidaria deviniendo posteriormente en un gran frente de masas.

El FER-SM aparece en los cincuenta en una lucha reivindicativa propiamente estudiantil, reformista, ante una universidad oligárquica que había en ese tiempo. Las convulsiones mundiales muestran una polarización entre reformismo y revolución, entre partidos que realmente quieren una revolución y los que quieren una reforma... ${ }^{28}$

A lo largo de sus cincuenta años de existencia, ha enfrentado continuamente contextos diversos; desde 1958 a $1969^{29}$, al APRA enquistado en las universidades; de 1969 a 1979, contra el gobierno militar, y en los ochentas, contra las tendencias del reformismo burocrático de Izquierda Unida (PCP-U, PCP-PR, PCR, PUM, PSR, entre otras agrupaciones) y el militarismo de izquierda, llámese PCP-Sendero Luminoso y el MRTA. Durante los años noventa contra el Estado corporativo y la intervención

\footnotetext{
$27 \quad$ Juventud Popular.

28 FER-SM.

29. La expulsión de Luis A. Sánchez como rector de la UNMSM es el punto culminante de la hegemonía aprista en la universidad.
} 
militar y administrativa de las universidades publicas como la Universidad Nacional Mayor de San Marcos.

En los setenta, la izquierda empieza a ser hegemónica y ganar arraigo en las masas, pero el frente se ira escindiendo y aparecerán otras organizaciones. La ruptura y las escisiones del FER-SM en los setenta se deben a que algunos sectores pasan a ser juventudes comunistas de partidos organizados, por consiguiente correas de transmisión. Al respecto nos señalan:

El FER logra una hegemonía política, orgánica e ideológica dentro del movimiento estudiantil, particularmente en San Marcos, que se va a prolongar durante toda la década del setenta. Durante esa década y en las subsiguientes se va dando varias subdivisiones dentro del FER. Inicialmente había sido un organismo de masas de carácter de frente único y después se fue subdividiendo en relación a los grupos de izquierda a nivel nacional.

[...] el FER en la práctica era un grupo de izquierda estudiantil marxista, pero conservo, digamos, la dinámica del frente único que no se oponía a la participación de los estudiantes, que no tenían una militancia partidaria, nunca se exigía eso. Eso lo distinguía de otras organizaciones que eran en realidad brazos juveniles de partidos políticos ${ }^{30}$.

Es en la década del setenta que el movimiento estudiantil sanmarquino logra su mayor desarrollo desde sus organismos gremiales, liderados por Frente Estudiantil Revolucionario. Es importante señalar el rol político estudiantil que cumplieron, Unidad Estudiantil, Vanguardia Revolucionaria y el PCP-PR, estos dos últimos, a fines de los setenta, serán las fuerzas predominantes, logrando un equilibrio político frente al FER-SM. Por esos años se establece una nueva relación y dinámica con el movimiento popular.

En el desarrollo del movimiento estudiantil, si algo ha caracterizado al FERSM, es la política de Frente Único. El frente se entiende dentro de la teoría marxista, como la puesta en práctica de la unidad para la acción de los sectores progresistas y revolucionarios frente a un determinado problema o ante la amenaza política del enemigo común. José Carlos Mariategui, señala que el frente no anula la personalidad política. Su concreción no es confusión ni amalgama, es una acción concreta que lo establece un programa ante una realidad inmediata. ${ }^{31}$ Sobre la importancia de una política de frente nos señalan:

\footnotetext{
30 FER.SM.

31 MARIÁTEGUI, José Carlos: Ideología y Política. Lima, Editorial Amauta, 1984, pp. 108-109.
} 
El frente único es una táctica política para luchar contra un enemigo más fuerte. Se forma sobre la base de un programa común a todos los que la conforman. Si no hay programa no hay frente. Es una unidad para la acción. Se basa en un programa y sirve para llegar a puntos concretos. Una vez conseguido los puntos que los une, el frente deja de ser, eso si no se revitalizan los puntos de unidad ${ }^{32}$.

d) El Colectivo Amauta y la construcción socializada del poder

El Colectivo Amauta surge el año 1997 impulsado por estudiantes de base y núcleos diversos, que al igual que Integración Estudiantil y Juventud Popular aspiran a convertirse en los nuevos referentes de la izquierda universitaria para generar espacios propios de liderazgo en la política estudiantil. Si bien se trata de una organización nueva, sus mentores y cuadros ideológicos tuvieron en la universidad, una significativa presencia a nivel docente, algunos militantes de partido políticos como el PCR (Partido Comunista Revolucionario) y el PUM (Partido Unificado Mariateguista), que durante los ochenta formaron parte de la Alianza Política Izquierda Unida. El Colectivo Amauta, agrupó fundacionalamente a organizaciones culturales y políticas como; el Taller de Estudios Sociales (TALES V), Democracia Estudiantil de la Facultad de Derecho y estudiantes de la tendencia anarquista y cultural de la Facultad de Letras, estos últimos, ligados al canto, la poesía, la trova y la música subte. Tuvieron una base estudiantil importante en la Facultad de Ciencias Sociales:

Ha sido un núcleo de derecho que disminuyó y gente de sociales, y algo en menor cantidad, de letras y arte. La gente de Derecho venía de Democracia Estudiantil. La gente, no toda [...] al menos yo no, de Sociales venía del TALES V, la de Letras venía más de la onda artística, culturosa ${ }^{33}$.

El Colectivo Amauta, tiene una estimulación inicial en la movida estudiantil de mayo y junio de 1997. Conciben la política de manera alternativa, donde la estrategia de emancipación frente al capitalismo ya no pasa necesariamente por la lucha de clases, sino por otras variables sociales como la horizontalidad del poder, la cuestión étnica, de género, la discusión del Estado y la nación. Ideológicamente han insertado a otros teóricos e intelectuales a la discusión clásica del anticapitalismo. Señalan la intención de desarrollar un nuevo discurso de izquierda. Ante esta realidad amorfa, se definen como socialistas. Rescatan a Marx, Lenin, Gramsci, Mariátegui, Marcuse, Proudhon y Bakunin, entre otros. Señalan no tener jefes. Proponen el poder desde abajo, fomentan la democracia radical, el autogobierno y la autogestión en la

\footnotetext{
32 FER-SM.

33 Colectivo Amauta.
} 
universidad, en los municipios y gobiernos regionales. Es una izquierda que ha girado más hacia la socialdemocracia. Aceptan lo lúdico como un aspecto de la política. Para ellos la política no está exenta de alegría ${ }^{34}$.

\section{Cuadro 1}

\begin{tabular}{|l|l|}
\hline Organizaciones políticas & Las organizaciones políticas en la UNMSM 1995-2000 \\
\hline Integración estudiantil & $\begin{array}{l}\text { Se forman el año } 1998 . \\
\text { Los problemas de la educación lo entendemos dentro de un } \\
\text { problema político mayor. } \\
\text { Nacimos como una organización de izquierda que se afirma } \\
\text { por el socialismo, pero que discrepa con los métodos de la } \\
\text { izquierda tradicional. }\end{array}$ \\
\hline Juventud popular & $\begin{array}{l}\text { Nos formamos en } 1995 . \\
\text { Nos definimos como un movimiento amplio, nacional, demo- } \\
\text { crático, patriótico y progresista. } \\
\text { Somos una organización que no solo trabaja a nivel estudian- } \\
\text { til, sino que nos proyectamos hacia otros sectores de la pobla- } \\
\text { ción como; el campesino, el obrero y el movimiento barrial. } \\
\text { La lucha contra el fujimorismo fue una de sus principales ban- } \\
\text { deras durante la segunda mitad de los noventa. }\end{array}$ \\
\hline FER-SM & $\begin{array}{l}\text { En 1958 se funda como Frente Estudiantil Revolucionario } \\
\text { en lucha abierta contra el APRA en San Marcos y por la de- } \\
\text { fensa de los derechos estudiantiles. } \\
\text { Hasta 1969, no se producen escisiones importantes. } \\
\text { Asumir la política de Frente Único como criterio orgánico. }\end{array}$ \\
\hline Colectivo Amauta & $\begin{array}{l}\text { Se formaron en 1997. } \\
\text { Fue un núcleo importante de compañeros de las Facultades } \\
\text { de Derecho que provenían de Democracia Estudiantil, estu- } \\
\text { diantes de la Facultad de Ciencias Sociales que venían del TA- } \\
\text { LES V, independientes de izquierda y gente de la Facultad de } \\
\text { Letras y Ciencias Humanas. }\end{array}$ \\
\hline
\end{tabular}

Fuente: Trabajo de campo

Elaboración: Propia

34 PAREDES Martín: “San Marcos: otras voces otros ámbitos”, en: Revista Quehacer N. 122, Desco, Lima, 1999. 


\section{Los acontecimientos}

Los acontecimientos están marcados por la dinámica universitaria y la coyuntura política nacional, que motivan una respuesta por parte del estudiantado y de sus organizaciones. Son los debates internos, las elecciones gremiales, las marchas por el cese de la comisión reorganizadora, las respuestas a las políticas elitistas y privatizadoras de la Universidad y las movilizaciones en el plano popular, los hechos más importantes durante el periodo 1995-2000. Como lo indicamos, es la época en que las prácticas políticas prevalecen sobre lo ideológico:

Encontré elecciones estudiantiles que en el caso de la facultad (derecho) fueron básicamente en torno al Centro Federado. El Gremio cumplió un papel adecuado en este escenario. Entonces la política para mí empieza a nacer en esta facultad. Encuentro un gremio luchando por los derechos estudiantiles, un espacio de gente peleando por erradicar las tendencias privatizadoras de la Universidad

Respecto a las movilizaciones recuerda:

... la primera en que yo participé aquí en la Universidad, fue a raíz de una huelga de hambre que había en ese entonces, por la reposición de estudiantes que habían sido expulsados por la comisión interventora, y luego una movilización que se dirigió de aquí, de la Universidad hacia el Congreso. Allí estaban discutiendo el tema de la intervención, que al final de cuentas se decidió que continuase. Luego recuerdo más las movilizaciones que se dieron con afluencia de los otros sectores de la sociedad, en el caso de los trabajadores, organizaciones ya no estudiantiles, sino sociales y gremiales. Dentro de ellos recuerdo el ingreso a palacio de gobierno en 1998. Fue algo que marcó, y estaba marcando el cambio de correlación que se estaba dando para enfrentar a la dictadura fujimorista y que llegó a ese nivel de expresión. En esas movilizaciones el respaldo estudiantil fue masivo. Recuerdo que mi base se organizó para eso. En ese tiempo el nivel de politización contra la dictadura fujimorista llegó a un nivel, por lo menos en esta facultad, de que las bases se organizaban y se pronunciaban sobre el problema y decidían participar en algunos casos, no en todos. Recuerdo eso y las últimas marchas que se dieron en el año 1999 y 2000, que fueron las que marcaron el quiebre y la derrota de un gobierno, de una dictadura cívico-militar, que fue la de Fujimori. Hubo también una movilización estudiantil que fue el 25 de mayo del 2000. Fue una movilización de casi todo el día. Muchos estudiantes en la mañana nos movilizábamos a pie, hubo enfrentamientos con la policía, llegamos a la Plaza de Armas, nos reprimieron, fuimos a canal 4 a protestar, porque canal 4 , como la prensa en todos los tiempos se ha 
acomodado con los que tiene el poder. Regresamos por el zanjón. Fue una movilización bastante larga"35.

Las fricciones con los militares eran comunes en los años que anteceden a la Comisión Reorganizadora (1990-1995) y los tres primeros años de su gestión (19951998). Un destacamento del ejército, acantonado en el Comedor Universitario, tenía la misión de resguardar el orden y la seguridad interna por mandato expreso del gobierno $^{36}$. Los estudiantes se movilizaban y en algunos casos estas manifestaciones culminaban en enfrentamientos con la policía en las intersecciones de las avenidas Venezuela y Universitaria, como ocurrió en Mayo (Instalación de la Camisón Reoganizadora), octubre de 1995 (reinauguración del Estadio de San Marcos por el gobierno) y marzo de 1996 (aumento del costo de la matricula). El seguimiento por parte de los militares y el espionaje de inteligencia hacia los estudiantes fue constante. Hubo muchas movilizaciones que fueron verdaderas jornadas políticas, tanto internas como externas como nos los recuerda el dirigente de Juventud Popular:

... estábamos con la intervención militar y administrativa. Había fricciones con las fuerzas militares en San Marcos. El 96, cuando ingresé, hubo una movilización interna, en donde se produce un tiroteo en la Facultad de Letras. También hubo enfrentamiento con los militares en la entrada de San Marcos cuando salimos a una protesta. Había luchas de los estudiantes por la gratuidad de la enseñanza e impedir que se sigan elevando los costos. En el 97 se llevaron a cabo movilizaciones considerables hacia el Congreso para exigir el cese de la comisión interventora en la Universidad. El 97 hubo varias movilizaciones previas, al cumplirse dos años de intervención. En el 98 las organizaciones juveniles; Juventud Popular, Integración Estudiantil que recién aparecía, el Colectivo Amauta, menos el FER, participamos en las marchas juveniles que hubo. Acá se impulsó la movilización del 4 y 11 de junio. Luego la marcha del 17 de julio.

El 4 de junio hubo 2000 estudiantes, el 11 éramos 3000 a 3500 estudiantes en las calles. Luego el 23 de junio realizamos una marcha por la resistencia. El 30 de septiembre hubo también una movilización que terminó con el ingreso de la gente al patio de Palacio de Gobierno, y a partir de ese entonces se dio toda una serie de luchas contra la dictadura.

$35 \quad$ Integración Estudiantil.

36 El 21 de mayo de 1991, el gobierno realiza visitas a las principales universidades públicas de Lima con el fin de constatar su real situación. El presidente de la República, la comitiva, las Fuerzas Armadas y Policiales que lo acompañaron bajo el pretexto de pintar las fachadas, son recibidos con una enérgica protesta y violencia por parte de los estudiantes sanmarquinos,...el gobierno la interviene militarmente. 
Luego el 99 fue el paro nacional del 28 de abril que convocaron los frentes regionales. Aquí se realiza un paro estudiantil y algunos estudiantes y jóvenes fuimos a bloquear las calles en la madrugada, a bloquear en los conos. [...]. El 5 de abril del 99 hicimos una pequeña marcha, una protesta por el autogolpe. Estábamos en unidad con todos los sectores, incluso los de derecha. En el 2000; las marchas del 25 de mayo y 8 de junio, donde hubo enfrentamientos con la policía. En noviembre, en la Facultad de Ciencias Sociales se da una toma para exigir que se vaya el decano ${ }^{37}$.

Para los militantes de Juventud Popular uno de los acontecimientos más significativos son las movilizaciones del 4 y 11 de junio de 1998, donde participaron activamente, tanto en la organización, desarrollo y balance, conjuntamente con otras organizaciones estudiantiles, asociaciones, colectivos, grupos de escolares, sindicatos y partidos políticos. Movilización abierta en contra de la dictadura fujimorista, protesta generalizada en los sectores mayoritarios que convocó a un grueso sector de la población, pero que se vio fundamentalmente como una movilización universitaria y juvenil que despertaba del letargo político ${ }^{38}$.

Con la intervención militar en la Universidad en mayo de 1991, el autogolpe del 5 de abril de 1992 y la lucha por la opción del NO ante la nueva Constitución Política del Perú en octubre de 1993, es que se forja un sector inicial de oposición al gobierno de Fujimori. Desde el frente interno, las reivindicaciones más significativas serán el derecho del pueblo a la educación, la lucha por los servicios básicos en la Universidad y contra la comisión reorganizadora. Al respecto un dirigente nos señala:

\begin{abstract}
Cuando ingreso a la universidad era muy rica en eventos políticos, dinámica. Hubo bastantes hechos en ese tiempo; conferencias, presentaciones y marchas. Había de todo y de todos los matices políticos e ideológicos.
\end{abstract}

Hubo muchas movilizaciones. Sobre todo por la defensa de los comedores, contra el referéndum, contra la militarización de San Marcos, contra la intervención en San Marcos ${ }^{39}$.

Pero entender el problema universitario, pasa también por observar sus micro realidades, es decir, el discurrir en las facultades, aulas o pasillos que también nos permitirán formular conjeturas respecto a estos espacios y su relación con el conjunto. Es decir, considerar el lado académico y el futuro profesional en sus aulas, sin dejar de lado la participación política. El acontecimiento político más importante en la

\footnotetext{
$37 \quad$ Juventud Popular.

38 En aquel entonces los medios de comunicación calificaron la masiva movilización, como el despertar de la juventud.

39 FER-SM.
} 
década de los noventa no fue la expresión de la derecha y de la reacción, evidenciada en la presencia de los militares, ni en las acciones de la Comisión Reorganizadora, sino, que la juventud se encontraba en el letargo y carecía de un sentido del cambio. $\mathrm{Al}$ respecto nos explican:

\begin{abstract}
¿Cuándo ingresée, la extrema derecha, el ejército, la comisión interventora. Nadie creía que algo era posible. Era la sensación universal. Mira gente de historia,... en esta facultad es bien pendejo, tu vas a encontrar antropólogos racistas, antropólogos que justamente su estudio consiste en desmontar ese discurso de poder que es la raza y hay gente que es racista. Vas a encontrar sociólogos autistas, que no se interesan por la sociedad. Vas a encontrar historiadores sin sentido de cambio. Mis amigos me decían; pero Jesús si siempre ha sido así, esto no va a cambiar, cambiará el nombre, el régimen, pero esto no podrá cambiar. No tenían sentido diacrónico de que las cosas pueden cambiar, no brutal e incendiariamente, pero sí estratégicamente. ¿ंTe das cuenta?, y eso era lo que me llegaba. Para mí el acontecimiento político más importante en San Marcos el 94, 95, 96, no ha sido que el ejercito estaba aquí, ni el rollo que nos metía la derecha. El acontecimiento político más importante por primera vez en el mundo es que la gente estaba adormecida $a^{40}$.
\end{abstract}

Dentro de este contexto, el proceso de maduración política de los diversos sectores políticos y no-políticos de las sociedad y la forja de la unidad frente al régimen autoritario, vera sus primeros atisbos en las movilizaciones de 1997 y 1998, donde van a converger distintas organizaciones en la lucha contra el régimen fujimorista ${ }^{41}$. Incluso sectores de derecha que vieron afectados sus intereses. En el caso de los dirigentes universitarios, el discurso será confrontacional. Es preciso indicar que muchos de ellos no compartieron lo que se dijo en la critica periodística y en los sectores de la intelectualidad, cuando se señaló a esta protesta social como la movilización de los jóvenes, como si se tratara de un grupo humano general y amorfo, sin un sentido de propuesta o como si la juventud no tuviera reparos y discusiones a su interior. Es decir, se obvio la existencia de sectores socialdemócratas, comunistas, socialistas, anarquistas; grupos de tendencia humanista, que estuvieron solo unidos ante un Estado corporativo, de tendencia dictatorial y que aplicó un modelo económico neoliberal. La juventud que participó en aquel entonces fue antifujimorista y en esencia anticapitalista:

\footnotetext{
$40 \quad$ Colectivo Amauta.

41 Cabe destacar las movilizaciones realizadas en junio de 1997 por las universidades particulares contra la destitución de tres magistrados del Tribunal Constitucional. Si bien este acto no puede ser considerado como una reivindicación netamente popular, en un primer momento concita la atención de amplios sectores de la población. El problema político se evidenciara durante los dos años siguientes con la derrota final del régimen.
} 
... Para mí era un movimiento popular antineoliberal, aunque para muchos era simplemente antidictatorial, antifujimorista. A partir de allí había perfilado un sentimiento que luego lo cuadré; ¿Por qué soy antifujimorista?, porque soy anticapitalista; así de sencillo. Otra gente era antifujimorista porque era socialdemócrata. Eran antifujimoristas por que eran liberales, o porqué eran demócratas. Es anticapitalista así de sencillo. Creo que yo pude decir recién esa frase el año 2000 con claridad, pero lo sentía ya desde el 97. Me llegaba que dijeran de la juventud, como si no habría divisiones e intereses dentro de ella, como si no hubiese ideologías que al menos intuitivamente la estén ganando" ${ }^{42}$.

Los jóvenes que participaron activamente en la organización y movilizaciones de aquel entonces, tenían perspectivas profesionales, económicas, laborales y políticas. Muchos de ellos luego serán asimilados al sistema. Implícitamente se demostró que la juventud careció de una formación y conciencia política mayor de trasformación de la sociedad desde las organizaciones, como si se dio en las generaciones anteriores.

$42 \quad$ Colectivo Amauta. 


\section{Cuadro 2}

\begin{tabular}{|c|c|}
\hline Mes/Año & $\begin{array}{l}\text { Principales acontecimientos político-universitarios durante } \\
1995-2000\end{array}$ \\
\hline Mayo 1995 & $\begin{array}{l}\text { Ley } 26457 \text { con la cual se inicia el proceso de reorganización de las universi- } \\
\text { dades San Marcos y la Cantuta. } \\
\text { Durante este mes se suceden una serie de movilizaciones tanto internas } \\
\text { como hacia el rectorado, ubicado en el edificio Kennedy en Lima. Hay en- } \\
\text { frentamientos con la policía en la Cuidad Universitaria y el rectorado }\end{array}$ \\
\hline $\begin{array}{l}\text { Octubre } \\
1995\end{array}$ & $\begin{array}{l}\text { En la víspera de las elecciones municipales, se inaugura la remodelación del } \\
\text { Estadio Monumental de la universidad por el gobierno central a través de la } \\
\text { Presidencia de la República. Asisten el Presiente de la República, el Ministro } \\
\text { de la Presidencia y el candidato a la alcaldía por Lima Jaime Yoshiyama. } \\
\text { Se realizan movilizaciones en el exterior de la Universidad y al interior por } \\
\text { algo de tres horas con enfrentamientos abiertos con la Policía Nacional. La } \\
\text { participación es masiva de los estudiantes. }\end{array}$ \\
\hline Marzo 1996 & $\begin{array}{l}\text { Se incrementan los cobros de matrícula y una serie de conceptos académi- } \\
\text { cos por cerca de } 300 \% \text {. Hay movilizaciones de protesta interna y hacia el } \\
\text { rectorado. En la Facultad de Letras hay forcejeos con las Fuerzas Armadas, } \\
\text { que en ese tiempo estaban a cargo del control y la seguridad interna de la } \\
\text { Universidad. Hubo disparos, creando un clima de inestabilidad en el claus- } \\
\text { tro sanmarquino. }\end{array}$ \\
\hline Мayo 1996 & $\begin{array}{l}\text { Con motivo del primer año de intervención administrativa en la Universi- } \\
\text { dad, se producen movilizaciones hacia el rectorado que concluye con graves } \\
\text { enfrentamientos con la Policía Nacional. Hay detenidos y heridos y bienes } \\
\text { afectados (patrulleros e inmuebles). Por esos días el Congreso de la Repú- } \\
\text { blica prorroga por un año más el ejercicio de la Comisiones Reorganiza- } \\
\text { dora. En semanas previas y posteriores se producen coordinaciones y otro } \\
\text { tipo de actos de protesta. }\end{array}$ \\
\hline Мayo 1997 & $\begin{array}{l}\text { A dos años de intervención administrativa nos movilizamos al Congreso } \\
\text { de la República. Se decide prorrogar por tiempo indefinido las comisiones } \\
\text { reorganizadoras. Previo a ello, algunos estudiantes inician una huelga de } \\
\text { hambre en la Universidad, teniendo el respaldo de sus compañeros y de } \\
\text { dirigentes políticos. }\end{array}$ \\
\hline Junio 1997 & $\begin{array}{l}\text { Tres magistrados del Tribunal Constitucional son destituidos por el Con- } \\
\text { greso de la República en clara maniobra política por la inaplicabilidad de } \\
\text { la llamada ley de Interpretación Auténtica. Se organizan en un primer mo- } \\
\text { mento jóvenes de universidades particulares y luego participan las universi- } \\
\text { dades nacionales. Se dan movilizaciones hacia la sede del poder legislativo. }\end{array}$ \\
\hline Junio 1998 & $\begin{array}{l}\text { Este mes quizá marca políticamente a muchos de mi generación. El } 4 \text { y } 11 \\
\text { de junio serán días recordados como el punto de quiebre por parte de los } \\
\text { jóvenes respecto a la llamada generación perdida, la década de la antipolítica } \\
\text { y la generación X. Esos días miles de jóvenes aproximadamente entre } 5 \text { mil } \\
\text { y } 10 \text { mil en su mayoría universitarios, protestan contra la política universita- } \\
\text { ria, educativa y general del gobierno. Las movilizaciones empiezan pasado } \\
\text { el mediodía y culminan cerca de las ocho de la noche. }\end{array}$ \\
\hline
\end{tabular}




\begin{tabular}{|c|c|}
\hline Mes/Año & $\begin{array}{l}\text { Principales acontecimientos político-universitarios durante } \\
1995-2000\end{array}$ \\
\hline Julio 1998 & $\begin{array}{l}\text { La CGTP convoca a una movilización donde participan universitarios. Re- } \\
\text { corren las principales avenidas de la ciudad y llegan a Palacio de Gobierno. }\end{array}$ \\
\hline $\begin{array}{l}\text { Septiembre } \\
1998\end{array}$ & $\begin{array}{l}\text { Se dan movilizaciones en Lima y en diferentes puntos del país. En Lima, un } \\
\text { grupo de trabajadores afiliados a la CGTP y al SUTEP, así como estudian- } \\
\text { tes de la Universidad San Marcos y otras universidades, ingresan al patio } \\
\text { principal de Palacio de Gobierno poniendo en vilo la vida de las personas y } \\
\text { la estabilidad política, pues hay una reacción casi inmediata, inusual y poco } \\
\text { razonada de las fuerzas del orden. Hay saqueos, enfrentamientos y disparos. } \\
\text { Otro día de jornada política que formó a los estudiantes. }\end{array}$ \\
\hline $\begin{array}{l}05 \text { abril de } \\
1999\end{array}$ & $\begin{array}{l}\text { Con motivo de conmemorase un año más del autogolpe de } 1992 \text { se pro- } \\
\text { ducen movilizaciones que recorren las calles del centro de Lima. Participan } \\
\text { estudiantes de San Marcos. }\end{array}$ \\
\hline Abril 1999 & $\begin{array}{l}\text { El régimen cada vez se hace insostenible. La CGTP convoca a un paro } \\
\text { nacional. Es acatada parcialmente, pero se ve el apoyo de otras fuerzas } \\
\text { políticas que antes no habían respaldado las jornadas de lucha convocadas. } \\
\text { En la cuidad universitaria hay bloqueos y enfrentamientos con la policía, a } \\
\text { la vez que se decreta un paro estudiantil. Hubo movilizaciones durante todo } \\
\text { el día por el centro de Lima. }\end{array}$ \\
\hline $\begin{array}{l}\text { Diciembre } \\
1999\end{array}$ & $\begin{array}{l}\text { El Jurado Nacional de Elecciones acepta la inscripción de la candidatura de } \\
\text { Alberto Fujimori. Se movilizan el pueblo, los sindicatos y universitarios en } \\
\text { señal de protesta. }\end{array}$ \\
\hline Abril 2000 & $\begin{array}{l}\text { Ante la posibilidad de un fraude electoral y el intento por tercera vez de } \\
\text { una reelección presidencial, los universitarios y el pueblo en su conjunto se } \\
\text { moviliza contra tal amenaza. Los sectores sociales estaban en unidad frente } \\
\text { al enemigo común de ese entonces: Fujimori y Perú } 2000 \text {. }\end{array}$ \\
\hline Mayo 2000 & $\begin{array}{l}\text { San Marcos participa con su pueblo para derrotar a la dictadura. Se suceden } \\
\text { movilizaciones durante todo el mes. }\end{array}$ \\
\hline $\begin{array}{l}\text { Noviembre } \\
2000\end{array}$ & $\begin{array}{l}\text { Dada la ley } 27366 \text {, cesan las comisiones reorganizadoras en las universi- } \\
\text { dades del país. Se inicia un nuevo periodo en la dinámica universitaria y } \\
\text { nacional. }\end{array}$ \\
\hline
\end{tabular}

Fuente: Trabajo de campo

Elaboración: Propia

\section{Logros de las organizaciones}

Los logros alcanzados por las organizaciones políticas esta en función de la evaluación de los discursos y acciones frente a los problemas universitarios y nacionales por parte de la comunidad estudiantil. Responden por su naturaleza, a formas de trabajo colectivo, que se legitiman con el objeto de afianzarse orgánicamente o de quedar 
relegado e incluso desaparecer de la vida política. Es el resultado de sus acciones lo que forja la identidad y se legitima en el tiempo, conservando la memoria de cada una de ellas.

En Integración Estudiantil se reafirman en haber contribuido a articular las luchas estudiantiles, el esclarecimiento de las bases y la formación de un espacio, donde la juventud universitaria opte por una posición de izquierda:

Bueno, en primer lugar; contribuir a la articulación de las luchas estudiantiles. Contribuir digo, porque no hemos sido la única organización. Han tenido su papel, incluso grupos de estudiantes que no han llegado a tener un nivel de organización política, pero como grupos estudiantiles tuvieron una posición y una actividad. Segundo; contribuir al esclarecimiento de los problemas estudiantiles. Tercero; el de brindar un nuevo espacio político a los estudiantes que están afirmándose por una nueva propuesta de izquierda. Entonces, me parece que ese espacio que hemos tratado de construir, ha tenido sus logros, se han ido agrupando compañeros para que se definan por la izquierda, en este caso por Integración Estudiantil ${ }^{43}$.

La lucha contra el Estado corporativo y la Comisión Reorganizadora en la Universidad Nacional Mayor de San Marcos, tuvieron desde el movimiento estudiantil una dispersa y frágil respuesta. Fue un acontecimiento trascendental para los dirigentes de Juventud Popular las movilizaciones del 4 y 11 de junio de 1998. Constituyó una de las jornadas de lucha de los noventa más importantes, donde los jóvenes insurgen contra el gobierno de Fujimori. Sin embargo no hubo una respuesta orgánica posterior a este trabajo inicial. De haberse dado, creemos, hoy la juventud universitaria estuviera institucionalizada como un organismo de presión valido en el escenario nacional:

Hemos sido contribuyentes de la concientización de un sector de los estudiantes, en la lucha contra la dictadura, contra la intervención en San Marcos. Apoyar para que la gente se concientice acerca de la realidad. En la organización gremial, de algunos centros de estudiantes y centro federados. Hasta ahora no hay un logro, salvo el 4 de junio que lo impulsamos con todo. Eso sirvió para que la gente despierte del letargo en la cual se encontraban, por el hecho de que ese día marcharon miles de San Marcos. Seria el haber contribuido a la derrota del fujimorismo y la intervención ${ }^{44}$.

\footnotetext{
$43 \quad$ Integración Estudiantil.

44 Juventud Popular.
} 
El FER-SM considera como logros, el haber mantenido el espíritu revolucionario en los jóvenes frente a las ideas reformistas y la socialdemocracia existentes en la universidad. El haber mantenido la política de frente único en las luchas por las reivindicaciones estudiantiles conquistadas en los años setenta, no por esta agrupación específicamente, sino en merito a todo un trabajo del movimiento estudiantil clasista de aquella época:

Uno de los principales logros en la actualidad, es el haber mantenido el espíritu de frente único en el estudiantado, el avance programático de la revolución en el frente cultural y el esclarecimiento para que no caigan en la social democracia, el militarismo y el conformismo. Mantener las luchas, las ideas desde la perspectiva revolucionaria. $\mathrm{Ha}$ habido logros anteriores que hasta ahora se mantienen, pero todos esos logros ahora están impedidos, todos los derechos que se ganaron con el FER, los defendimos durante los setenta y los ochenta. Esos derechos que están hoy impedidos, hay que tratar de reconquistarlos y defenderlos. Pero no quiero hablarte de los logros del FER, sino del logro de todo el estudiantado, cuando había un frente único verdaderamente fuerte en la universidad. Han sido los logros de todo el estudiantado sanmarquino de aquel entonces ${ }^{45}$.

Haber contribuido al debate de nuevas propuestas anticapitalistas no necesariamente marxistas-leninistas desde la horizontalidad democrática del poder y la generación de un espacio político alternativo en la universidad son los logros alcanzados por el Colectivo Amauta:

Primero; haber construido al menos en el colectivo, una política democrática horizontal. Es decir, entre nosotros al menos hay relaciones comunistas. Me refiero en el sentido que no hay poder, todos tenemos igual poder. Alguien puede tomar la iniciativa, meter pilas, pero nadie manipula a nadie, uno puede influir en el otro; es cierto, pero nadie instrumentaliza a nadie. Segundo; que la producción de ideas hemos ido clasificándolas. Tercero; si bien no hemos logrado construir un sentido común, anticapitalista como nos agradaría, si hay gente que puede ser bacán para este proyecto, que puede tener potencialidades, descubrirla ¿no? y pincharle a ver si actúan ${ }^{46}$.

\footnotetext{
45 FER-SM.

46 Colectivo Amauta.
} 


\section{Cuadro 3}

\begin{tabular}{|c|c|}
\hline Organizaciones políticas & Logros de la organización \\
\hline Integración estudiantil & $\begin{array}{l}\text { La articulación de las luchas estudiantiles. } \\
\text { Haber contribuido al esclarecimiento de los problemas } \\
\text { estudiantiles. } \\
\text { Haber brindado un espacio político a los compañeros } \\
\text { estudiantes y que estos se definan por la izquierda. }\end{array}$ \\
\hline Juventud Popular & $\begin{array}{l}\text { Hemos contribuido a la concientización de un sector } \\
\text { del estudiantado, a la lucha contra la intervención en } \\
\text { San Marcos y la dictadura. } \\
\text { Impulsamos la movilización del } 4 \text { y } 11 \text { de junio de } 1998 .\end{array}$ \\
\hline FER-SM & $\begin{array}{l}\text { El haber mantenido el espíritu de Frente Único en la } \\
\text { Universidad y el avance programático de la revolución } \\
\text { en el frente cultural. } \\
\text { Al esclarecimiento, para que los estudiantes no caigan } \\
\text { en la socialdemocracia, el militarismo y el conformismo. } \\
\text { Los derechos estudiantiles fueron conquistados en la } \\
\text { década del setenta por todos los estudiantes }\end{array}$ \\
\hline Colectivo Amauta & $\begin{array}{l}\text { El haber construido un poder democrático horizontal. } \\
\text { La producción de ideas hemos ido clasificándolas. } \\
\text { La construcción de un proyecto anticapitalista alterna- } \\
\text { tivo. }\end{array}$ \\
\hline
\end{tabular}

\section{Conclusiones}

-El estudiante de la los noventa tiene perspectivas individuales antes que colectivas. El discurso neoliberal se integra en él no solo a nivel político, sino que inconscientemente influye en su persona, de tal manera que es un nuevo tipo de estudiante que difiere al de décadas anteriores. Los mitos y las ideologías son remplazadas por las certezas. La realización personal y profesional se logra en el presente a través de la práctica cotidiana.

- Los cambios y las continuidades de las organizaciones, siguen siendo importantes, en la medida que los discursos y prácticas inciden en primer lugar a reconstruir una historia particular, la historia política de la izquierda en la Universidad de San Marcos en los noventa, y segundo; mostrar el proceso y constitución de estas nuevas identidades como alternativas. Ellas surgen a partir de mediados de los noventa, a excepción del FER-SM que es una organización estudiantil con cincuenta años de existencia. Son los años de la consumación y el descrédito de la política y de la izquierda en particular. A pesar de ellos creemos, existieron ideas y propuestas desde el socialismo en la universidad. 
- La articulación del movimiento estudiantil con el movimiento popular, el esclarecimiento de las bases, los espacios de discusión y propuestas, el frente único y el programa de cambio social, así como el haber desarrollado espacios democráticos horizontales hacia la construcción de un proyecto anticapitalista, son los logros significativos de las organizaciones durante la segunda mitad de los noventa. La perspectiva socialista no está ausente, al menos en la vanguardia estudiantil, es decir, en el sector más politizado de los estudiantes.

\section{BIBLIOGRAFÍA}

BEBBINGTON, Anthony, Martín; SCURRAH y Claudia BIELICH

2008 Mapeo de Movimientos Sociales en el Perú Actual. Lima, CEPES.

BERNALES, Enrique

1974 Movimientos sociales y movimientos universitarios en el Perú. Lima, PUCP.

CASTELLANOS, Themis; CARAMUTTI, Rosalía y UBILLUS, Mercedes

2003 Lo viejo y lo nuevo de la acción colectiva en Lima: experiencias de participación ciudadana en Lima, Centro Alternativa, Lima.

CHÁVEZ, Jorge

1998 Los Universitarios y su Participación en la Vida Politica Nacional. Lima, Agenda-Perú.

GROMPONE, Romeo

1991 El velero en el viento. Sociedad y política en Lima. Lima, Instituto de Estudios Peruanos

KRAUSKOPF, Dina:

2000 "Dimensiones críticas en la participación social de las juventudes", en Sergio BALARDINI, La participación socialy politica de los jóvenes en el horizonte del nuevo siglo. Buenos Aires, CLACSO.

LYNCH, Nicolás

1990 Los jóvenes rojos de San Marcos. Lima, El zorro de abajo.

MALPARTIDA, Víctor y HOCES, Luis

1992 Encontrando el horizonte utópico. La esperanza jodida de los jóvenes. Lima, Grafi Perú EIRL.

MARIATEGUI, José Carlos

1970 Temas de Educación. Lima, Editorial Amauta.

1984 Ideología y Politica. Lima, Editorial Amauta.

MEJÍA, Carlos

1995 "Silencios y sonrisas en los pasadizos", en: Herejes y Renegados, segunda

MIRANDA, Oscar etapa, N. ${ }^{\circ} 14$, agosto-septiembre, Lima.

2002 "San Marcos. Detrás del Che se esconde Mao. Radicales en la sombra”, en: Domingo, La República, N. ' 213, Lima, 30 de junio de 2002. 
MONTOYA, Luis

1992 “Las otras elecciones. San Marcos: la otra mayoría”, en: Revista Quehacer, N“80, Desco, Lima, 1992.

1993 Nido de inquietudes. Universidades y jóvenes: politica de desarrollo universitario, actores sociales y modernidad educativa, Tesis para optar el Título Profesional de Licenciado en Sociología, Lima, UNMSM, 1993.

1994 "Los varios colores de la juventud universitaria", en: Revista de Sociología, Vol. 8, N” 9, Lima, UNMSM.

PAREDES, Manuel:

1999 Mensaje del Rector al Estudiante Sanmarquino e Informe del Proceso de Reorganización de la Universidad Nacional Mayor de San Marcos, Mayo 1995 a marzo 1999. Lima, Fondo Editorial UNMSM.

1999 "San Marcos: otras voces otros ámbitos", en: Revista Quehacer N." 122, Desco, Lima.

2000 Memoria 1995-1999. Lima, Fondo Editorial, UNMSM.

PONCE, Grover

$\mathrm{s} / \mathrm{f} \quad$ El discreto encanto del desencanto. Jóvenes, politica y régimen fujimorista en San Marcos de los noventa, Mimeo, Lima.

POOLE, Deborah y RENIQUE y Gerardo

2001 "Movimiento popular, transición democrática y caída de Fujimori”, en: Memoria 147, México.

VARGAS, Moisés

2002 La violencia política en la escena sanmarquina: 1990-2000, mimeo, Lima. 